\title{
Addressing racism and disparities in the biomedical sciences
}

\author{
Bias and racism in the biomedical community thwart scientific advancement, reduce the pipeline of diverse \\ clinicians and scientists, and contribute to racial and ethnic health disparities. We advocate for proactive \\ antiracism approaches to eliminate barriers impacting people of colour, promote equity and achieve a more \\ effective biomedical community.
}

Uraina S. Clark and Yasmin L. Hurd

\section{An unprecedented time that reveals our past}

COVID-19 has pulled back the curtain on the stark health disparities and inequities in the US. From the very early months of the pandemic, gravely disparate outcomes have been reported for people who are Black, Indigenous or people of colour (BIPOC). For example, in New York City, Black and Latinx citizens were twice as likely to die from COVID-19 as white citizens. Sadly, these data confirmed the perceptions of BIPOC Americans, who were twice as likely as white Americans to consider the coronavirus a serious risk to their health even before the height of the pandemic in the US. They knew what health officials, politicians and their fellow white citizens did not: that they live in a different America, have different healthcare access and experience biases that frequently put them most at risk.

The COVID-19 pandemic, just months old, converged on the centuries-old racism pandemic that inflicts the greatest health risks upon BIPOC citizens. Health disparities and inequities in the US stem from multiple root causes, including poverty, poor access to gainful employment, exposure to environmental toxins, unhealthy environmental conditions in BIPOC and low-income communities, unhealthy housing, unhealthy work conditions, and the concentration of disadvantage among specific groups of people and in specific locations ${ }^{1}$. However, implicit biases also play a major role ${ }^{2}$, as it has been documented that BIPOC patients often receive a lower quality of care relative to their white counterparts, even after controlling for confounding factors (for example, insurance status, socioeconomic status, severity of illness, comorbidities $)^{3}$.

Disparities in the biomedical sciences Combating health disparities is complex by the very nature of its root causes but most appear to agree that it requires the development of a diverse biomedical workforce. Greater diversity is associated with greater patient participation in care, higher patient satisfaction, greater patient adherence to treatment, and greater reach into BIPOC and medically underserved communities $^{3}$. Indeed, a diverse biomedical workforce includes not only clinicians, but also translational researchers and basic science investigators. Teams with greater diversity have been shown to develop more effective solutions and publish higher impact papers than non-diverse teams ${ }^{4}$. BIPOC scientists contribute novel research perspectives and thus help to advance research innovation. Yet, despite the many strengths that BIPOC faculty offer, achieving diversity in the US biomedical workforce remains a major challenge.

BIPOC faculty continue to be underrepresented in clinical and research-related professions and are less likely to secure federal funding than white faculty ${ }^{5}$. Even with comparable measures of scientific achievement (for example, previous grants, publications), BIPOC investigators have lower levels of success in obtaining NIH funding ${ }^{6}$ and attaining high-level promotions within their academic institutions relative to their white peers. There is a concrete ceiling. As a result of the continued paucity of BIPOC investigators and leaders in academic medicine, BIPOC students and junior investigators have fewer culturally sensitive mentors, which in turn contributes to a very narrow entry into a 'leaky pipeline', as without this support many drop out or do not even attempt the academic journey.

\section{Impaired by bias}

For far too many BIPOC scientists and practitioners, working in an environment in which they feel unseen, unheard, unsupported and unwelcomed is a key barrier to success. A recent survey of university faculty found that BIPOC and female faculty "feel they have to work harder than their colleagues to be perceived as a legitimate scholar," with approximately a third of male and more than half of female BIPOC faculty reporting that they experience stress due to discrimination. Such data accord with prior reports indicating high rates of workplace discrimination for BIPOC faculty and students in academic medicine. Those who experience discrimination and/ or abuse are more likely to report burnout than those in academic medicine who have not experienced this mistreatment ${ }^{8}$. Discrimination is also associated with a higher turnover rate for BIPOC faculty, as well adverse psychological, neural, physiological and medical outcomes? Furthermore, exposure to discrimination, and even observing subtle biased acts against others, negatively impacts cognitive performance $^{10}$. Thus, not surprisingly, for those BIPOC scientists and clinicians who stay the course, success comes at significant cognitive, mental health and physical costs.

Similarly to BIPOC Americans who knew that they would suffer the most from the COVID-19 pandemic, BIPOC students and scientists know that they have a much steeper hill to climb in academia due to racism and bias. We queried some of our BIPOC colleagues around the nation about experiences of bias and discrimination. Some of these experiences are included, with permission, in Box 1. Several colleagues recounted experiences of being considered intellectually inferior, invalidated, subject to abuses of power, in unstable relationships with colleagues, excluded from or overlooked for opportunities, isolated, viewed as an outsider and viewed as a commodity. Such experiences serve to impede the advancement of our BIPOC 
Box 1 | Sample vignettes experienced by BIPOC peers in science and academic medicine reflecting bias and lack of sensitivity in the environments and institutions in which they train and work

- My colleague told me that health disparities research "is not science."

- My colleague told me that "Blacks have lower IQs than whites."

- My colleague told me that when we began working together, they questioned whether they wanted to continue working with me given my views about race. We never had any such conversations about race. This person was my direct supervisor for many years.

- My colleagues often mistake me for one of the other Black persons in our department. We are of different heights, hues, and builds.

- My colleague and fellow faculty member of over 5 years asked me to register a patient. I explained that I was not the receptionist. My colleague insisted, seeming to think that I was a receptionist from another unit.

- Throughout all of my education, it was not until graduate school that I had an underrepresented minority (URM) teacher/professor, and even here there were only two faculty of colour. I sometimes wonder how the absence of URM faculty impacts my colleagues' abilities to see minorities as experts.

- My colleague said to me, out of the blue, "We used to sell your people."

- My colleagues offer a constant stream of mixed messages defined by verbal support of minorities in science but actions that directly contradict this support. This system of tokenism through messaging situates minorities in an environment that is only superficially supportive while permitting subtle forms of discrimination that limit minority advancement. Working in such a system is exhausting.

- The director of my organization told me that, as a scientist, "you're not really Black because you are good."

- My supervisor told me, "I can't understand your language when you speak." I was born and raised speaking English, obtained an English minor degree, and was a nationwide radio presenter. I was also a senior faculty member at the time.

- My colleague, in the presence of our shared mentor, told me I didn't belong in our lab, implying that I was hired only due to the lack of diversity in our department. My mentor did not challenge this narrative.

- As the only Black faculty member in my medicine department, I was always 'voluntold' to intermediate with minority trainees; the assumption being that one minority's experience mirrors that of all others despite regional, socioeconomic and ethnic backgrounds.

- Numerous white colleagues have asked why I didn't apply for the "minority money" since in their view this is easier to get than grants from the general $\mathrm{NIH}$ K01 funding pool. They assume that African American scientists are less qualified and thus can only compete for funding designated for underrepresented populations. Unfortunately, this assumption often leads people to view these programs as handouts rather than seeing them for what they are: a means to fund exceptional scientists who are too frequently overlooked because of racism. colleagues and by extension science and medicine as a whole.

The biases that impact our BIPOC colleagues also collaterally impact health disparities for BIPOC patients. Recent data indicate that BIPOC investigators face greater difficulty, relative to their white peers, in obtaining NIH funding, due to the types of research areas many tend to gravitate towards, such as health disparities or community health research, which receive lower award rates 5 . Lower award rates for research areas in which BIPOC investigators are over-represented may be due, at least in part, to implicit bias, as decreases in the proportion of individuals from the majority culture in a given field are known to lead to decreases in the value ascribed to that field ${ }^{11}$. Considering the impact that health disparities have on communities of colour, as well as on our nation as a whole-a fact that has been glaringly exposed by the COVID-19 pandemic-we must address the possibility that racial and social biases play a role, not only in the assessment of BIPOC investigators $^{6}$, but also in the research area preferences that reviewers display. Bias and racism are founded on fallacy; decisions that are impaired by bias are antithetical to science and jeopardize our ability to adequately perceive and address some of the major health issues that we face as a nation and worldwide.

\section{New approaches to old problems}

The persistence of health and workforce disparities demonstrates the failure of 'colourblind' approaches to address racism and discrimination. Moving forward, more proactive 'race-conscious' or antiracism approaches are needed. Such approaches can provide (1) cognitive skillsets necessary to identify and critically analyze our biased assumptions and (2) psychological tools required to engage in healthy dialogues about bias, racism, structural inequalities and other social conditions (for example, poverty) that underlie health disparities in the US.

Indeed, several medical schools have begun to integrate these approaches into their curricula. It is, however, time for antiracism approaches to also become more widely adopted by faculty (Table 1). Many institutions have developed diversity programs designed to attract and foster BIPOC faculty. Considering the vital role that BIPOC investigators play in addressing health disparities, and in advancing science more generally, these programs are necessary and impactful. Yet, focusing only on BIPOC faculty is a piecemeal approach and does not directly address the real biases they face. Programs that provide all faculty and institutional leaders with the opportunity to engage with antiracism principles ${ }^{12}$ are needed. This training should not be a superficial checkbox. It should be fully integrated into our faculty development programs, as we know that while brief training programs can reduce implicit biases and unintended acts of discrimination, their effects diminish over time. In fact, all biomedical professionals and staff should receive regular training in the application of antiracism principles to more comprehensively mitigate our current climate of bias.

Beyond enhancing this core competency for all faculty and staff, antiracism principles should inform the creation of novel policies that advance equity in biomedicine. These policies should be coupled with systems of accountability that incentivize and track resulting gains in equity, as well as identify areas in need of improvement. Institutional resource allocations should be linked to measurable equity accomplishments.

Additional initiatives at the institutional level should include policies that ensure 


\section{Table 1 | Potential approaches to transition our current allegedly colourblind landscape towards a more proactive race-conscious or antiracist environment. Systems that support integrated efforts across national and institutional initiatives are required}

\section{Institutionally led initiatives}

Require implicit bias and antiracism training for all faculty, staff and leaders at academic institutions Create policies that are informed by antiracist principles to advance equity

Develop systems of accountability that incentivize and track equity successes, as well as areas of needed improvement

Promote equity gains by linking institutional and departmental resources to equity outcomes

Create policies that ensure equitable institutional resources to BIPOC and other underrepresented trainees and faculty

Ensure systems of promotion that value non-traditional service and contributions (for example, development of community outreach programs) on par with traditional contributions (for example, expansion of a clinical service)

Create policies that ensure non-science-related administrative duties are distributed equitably

Develop locally-tailored solutions through consultations with BIPOC trainees/faculty and with diversity, equity, and inclusion experts

\section{Nationally led initiatives}

Provide structured nationwide antiracism training for all teachers, from preschool to high school

Expand nationwide programs to provide middle and high school BIPOC students with early exposure to academic scientific environments

Develop initiatives that match BIPOC graduate students, Fellows and junior faculty with effective cultural role models and mentors across disciplines and institutions

Establish leadership training programs for BIPOC trainees and junior faculty

Develop sustained long-term funding mechanisms, both at the local and national level, to enhance diversity, inclusion and equity programs

equitable support of BIPOC and other underrepresented trainees and faculty (for example, access to resources, compensation, start-up packages, administrative support); systems of promotion that value 'non-traditional' contributions (for example, development of community outreach programs) on par with 'traditional' contributions (for example, expansion of a clinical service); and policies to ensure that non-science-related administrative duties are distributed equitably among all faculty. Additional locally tailored solutions should be derived through consultations with BIPOC trainees and faculty and with diversity, equity and inclusion experts, while maintaining an eye towards antiracism and intersectionality. Importantly, institutions must take responsibility for developing these programs, rather than placing the onus solely on BIPOC trainees and faculty.

Nationwide, innovative programs that intervene early, address inequities in training opportunities for BIPOC students and create nurturing environments that remove barriers to entry in academia must be devised. These may include nationwide programs that provide antiracism training for teachers and mentors from preschool onward and programs that engage BIPOC middle and high school students in structured scientific opportunities at higher academic institutions. These programs should be coupled with initiatives that elevate the voices of BIPOC trainees and faculty to address inequities in mentorship and visibility. These could include programs that match BIPOC graduate students, Fellows and junior faculty with cultural role models and mentors across disciplines and institutions, as well as programs that establish leadership training programs for BIPOC trainees and junior faculty. Importantly, we must continue to emphasize the true purpose of these initiatives, which is to counteract systemic racism, enhance equity and, most notably, recognize merit; this emphasis is essential, as it actively challenges the false yet enduring notion that these programs exist because BIPOC are inherently less capable-a notion that is rooted in racism.

Our culture of bias is deeply entrenched. In times of stress, as fully evident today, implicit biases can become stronger and more prominent ${ }^{13}$. Though recently many people have been rightly outraged by the brutal police murders of countless Black citizens and have galvanized global calls for equity in society, once the initial outrage subsides, discussions of equity often dissipate, due in part to fears that systemic change might involve the sharing of limited local resources. As such, we must engage national resources to adequately effect change. Systemic problems require systemic solutions. We must develop long-term funding mechanisms to support and sustain programs designed to combat the cultural norm of bias, as inconsistent implementation and funding jeopardize the gains made. A full investment in these programs, at both the local and national level, is necessary to ensure continued success. Indeed, all organizations should immediately examine their budgets to determine how proper allocation can enhance diversity, inclusion and equity. During times of fiscal hardship, like the one associated with the COVID-19 pandemic, institutions often resort to spending cuts. Programs will be assessed from many vantage points, with those considered nonessential being most at risk. If history is any indication, programs prioritizing diversity will be among those in jeopardy. However, now is the time in which these programs and efforts are needed most.

\section{Equity is a public good}

Eliminating health disparities constitutes a compelling public interest, as health disparities negatively impact individuals and communities, as well as the economy. While the COVID-19 pandemic has revealed the glaring magnitude of health disparities that exist in the US due to endemic racism, it has also once again highlighted disparities within the biomedical academic community that must be resolved. As a scientific community, it is urgent that we acknowledge the continued atmosphere of bias, which negatively impacts the capacity of BIPOC faculty and trainees and, ultimately, the care delivered to BIPOC patients. Overcoming this challenge will require sustained effort, coupled with a multisystem approach that re-envisions many of our policies, organizations and programs to better address those factors that contribute to disparities ${ }^{14,15}$. A key component to our success will be our individual and collective ability to recognize and eradicate racism and other biases-both in our own biomedical community and in the community at large-that sustain these inequities. Together, we can once and for all flatten the curves of these pandemics.

\footnotetext{
Uraina S. Clark iD 1,2,3凶 and

Yasmin L. Hurd (iD 2,4,5凶

${ }^{1}$ Department of Neurology, Icahn School of Medicine at Mount Sinai, New York, New York, USA. ${ }^{2}$ Addiction Institute of Mount Sinai, Icahn School of Medicine at Mount Sinai, New York, New York, USA. ${ }^{3}$ Center for Scientific Diversity, Icahn School of Medicine at Mount Sinai, New York, New York,
} 
USA. ${ }^{4}$ Department of Psychiatry, Icahn School of Medicine at Mount Sinai, New York, New York,

USA. ${ }^{5}$ Department of Neuroscience, Icahn School of Medicine at Mount Sinai, New York, New York, USA.

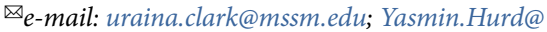
mssm.edu

Published online: 10 July 2020

https://doi.org/10.1038/s41562-020-0917-7

References

1. Centers for Disease Control and Prevention. CDC Health Disparities \& Inequalities Report - United States, 2013 https:// www.cdc.gov/MMWR/pdf/other/su6203.pdf (U.S. Department of Health and Human Services, 2013).

2. Hoffman, K. M., Trawalter, S., Axt, J. R. \& Oliver, M. N. Proc. Natl Acad. Sci. USA 113, 4296-4301 (2016).
3. Institute of Medicine. Unequal Treatment: Confronting Racial and Ethnic Disparities in Health Care (eds Smedley, B. D., Stith, A. Y. \& Nelson, A. R.) (National Academies Press, 2003).

4. AlShebli, B. K., Rahwan, T. \& Woon, W. L. Nat. Commun. 9 5163 (2018).

5. Hoppe, T. A. et al. Sci. Adv 5, w7238 (2019).

6. Ginther, D. K. et al. Science 333, 1015-1019 (2011).

7. Stolzenberg, E.B. et al. Undergraduate Teaching Faculty: the HER Faculty Survey 2016-2017. https://heri.ucla.edu/monographs/ HERI-FAC2017-monograph.pdf (Higher Education Research Institute, UCLA 2019)

8. Hu, Y. Y. et al. N. Engl. J. Med. 381, 1741-1752 (2019).

9. Williams, D. R., Lawrence, J. A., Davis, B. A. \& Vu, C. Health Serv. Res. 54(Suppl 2), 1374-1388 (2019).

10. Ozier, E. M., Taylor, V. J. \& Murphy, M. C. J. Soc. Issues 75, 1087-1115 (2019).

11. Levanon, A., England, P. \& Allison, P. Soc. Forces 88 865-891 (2009).

12. Came, H. \& Griffith, D. Soc. Sci. Med. 199, 181-188 (2018).
13. Johnson, T. J. et al. Acad. Emerg. Med. 23, 297-305 (2016). 14. Williams, D. R. \& Cooper, L. A. Int. J. Environ. Res. Public Health 16, 606 (2019).

15. Jones, C. P. Ethn. Dis. 28(Suppl 1), 231-234 (2018).

Acknowledgements

Y.L.H. is supported by NIH grants DA15446, DA050323 and DA030359. None of the funders had a role in the conceptualization of, preparation of, or decision to publish this manuscript. The views expressed in this article are those of the authors and do not necessarily reflect the position or policy of the NIH. The authors thank E. Benn for her helpful insights, and we thank our colleagues from around the nation who shared their personal experiences with us, which were all extremely valuable though some could not be cited.

Competing interests

The authors declare no competing interests. 